\title{
Semicommutators and Zero Product of Block Toeplitz Operators with Harmonic Symbols
}

\author{
Puyu Cui, ${ }^{1}$ Yufeng Lu, ${ }^{1}$ and Yanyue Shi ${ }^{2}$ \\ ${ }^{1}$ School of Mathematical Sciences, Dalian University of Technology, Dalian 116024, China \\ ${ }^{2}$ School of Mathematical Sciences, Ocean University of China, Qingdao 266100, China \\ Correspondence should be addressed to Yanyue Shi; shiyanyue@gmail.com
}

Received 15 February 2013; Accepted 17 August 2013

Academic Editor: Gen-Qi Xu

Copyright (c) 2013 Puyu Cui et al. This is an open access article distributed under the Creative Commons Attribution License, which permits unrestricted use, distribution, and reproduction in any medium, provided the original work is properly cited.

We completely characterize the finite rank semicommutators, commutators, and zero product of block Toeplitz operators $T_{F}$ and $T_{G}$ with $F, G \in h^{\infty} \otimes M_{n \times n}$ on the vector valued Bergman space $L_{a}^{2}\left(\mathbb{D}, \mathbb{C}^{n}\right)$.

\section{Introduction}

Let $\mathbb{D}$ be the open unit disk in the complex plane $\mathbb{C}$, and let $d A$ be the normalized Lebesgue area measure on $\mathbb{D}$. Denote by $L^{\infty}(\mathbb{D}, d A)$ and $L^{2}(\mathbb{D}, d A)$ the space of essential bounded measurable functions and the space of square integral functions with respect to $d A$, respectively. The Bergman space $L_{a}^{2}$ consists of all analytic functions in $L^{2}(\mathbb{D}, d A)$. Let $L^{2}\left(\mathbb{D}, \mathbb{C}^{n}\right)=$ $L^{2}(\mathbb{D}, d A) \otimes \mathbb{C}^{n}$ and $L_{a}^{2}\left(\mathbb{D}, \mathbb{C}^{n}\right)=L_{a}^{2} \otimes \mathbb{C}^{n}$, where $\otimes$ means the Hilbert space tensor product. Let $M_{n \times n}$ be the set of all $n \times n$ complex matrixes in the complex plane $\mathbb{C}$, and let $L^{\infty}(\mathbb{D}, d A) \otimes M_{n \times n}$ be the space of matrix valued essential bounded Lebesgue measurable functions on $\mathbb{D}$.

Given a matrix valued function $\Phi(z)=\left[\varphi_{i j}(z)\right]_{n \times n} \in$ $L^{\infty}(\mathbb{D}, d A) \otimes M_{n \times n}$, the Toeplitz operator $T_{\Phi}$ and the Hankel operator $H_{\Phi}$ on $L_{a}^{2}\left(\mathbb{D}, \mathbb{C}^{n}\right)$ with symbol $\Phi$ are defined by $T_{\Phi} h=P(\Phi h)$ and $H_{\Phi} h=(I-P)(\Phi h)$, respectively, where $P$ is the orthogonal projection from $L^{2}\left(\mathbb{D}, \mathbb{C}^{n}\right)$ onto $L_{a}^{2}\left(\mathbb{D}, \mathbb{C}^{n}\right)$. Then the operators $T_{\Phi}$ and $H_{\Phi}$ have the following matrix representations:

$$
T_{\Phi}=\left[\begin{array}{ccc}
T_{\varphi_{11}} & \cdots & T_{\varphi_{1 n}} \\
\vdots & & \vdots \\
T_{\varphi_{n 1}} & \cdots & T_{\varphi_{n n}}
\end{array}\right], \quad H_{\Phi}=\left[\begin{array}{ccc}
H_{\varphi_{11}} & \cdots & H_{\varphi_{1 n}} \\
\vdots & & \vdots \\
H_{\varphi_{n 1}} & \cdots & H_{\varphi_{n n}}
\end{array}\right] .
$$

The problem of the finite rank semicommutator of two Toeplitz operators on the Hardy space has been completely solved in $[1,2]$. The analogous problems on the Bergman space have been characterized in [3-5]. In the case of vector valued Hardy space, $\mathrm{Gu}$ and Zheng [6] have studied the problems of compact semicommutators and commutators of two block Toeplitz operators.

In this paper, we study the related problems of the two block Toeplitz operators $T_{F}$ and $T_{G}$ with $F, G \in h^{\infty} \otimes M_{n \times n}$ on the vector valued Bergman space $L_{a}^{2}\left(\mathbb{D}, \mathbb{C}^{n}\right)$, where $h^{\infty}$ denotes the space of all bounded harmonic functions on $\mathbb{D}$. Our main idea here is to reduce the problems on $L_{a}^{2}\left(\mathbb{D}, \mathbb{C}^{n}\right)$ to the problems of the finite sum of some Toeplitz operator products or some Hankel operator products on the Bergman space $L_{a}^{2}$. In the following, we recall two useful theorems. For every $f \in h^{\infty}$, denote by $f_{+}$(or $f_{-}=f-f_{+}$) the analytic part (or the coanalytic part) of $f$.

Theorem 1 (see [7]). Suppose that $f_{j}$ and $g_{j}$ are bounded harmonic functions on $\mathbb{D}$ for $j=1, \ldots, k$. Then the following are equivalent:

(1) $\sum_{j=1}^{k} H_{\bar{g}_{j}}^{*} H_{f_{j}}$ has finite rank,

(2) $\sum_{j=1}^{k} H_{\bar{g}_{j}}^{*} H_{f_{j}}=0$,

(3) $\sigma\left(f_{1}, \ldots, f_{k} ; g_{1}, \ldots, g_{k}\right) \equiv 0$,

where $\sigma\left(f_{1}, \ldots, f_{k} ; g_{1}, \ldots, g_{k}\right)=\widetilde{\Delta}\left[\sum_{i=1}^{k}\left(f_{i}\right)_{-}\left(g_{i}\right)_{+}\right]=(1-$ $\left.|z|^{2}\right)^{2} \sum_{i=1}^{k}\left(f_{i}\right)_{-}^{\prime}\left(g_{i}\right)_{+}^{\prime}$. 
Theorem 2 (see [8]). Let $u_{1}, \ldots u_{n}, v_{1}, \ldots, v_{n}$ be bounded harmonic functions on $\mathbb{D}$, and $x_{1}, \ldots, x_{n}, y_{1}, \ldots, y_{n} \in L_{a}^{2}$. Then $\sum_{j=1}^{n} T_{u_{j}} T_{v_{j}}=\sum_{j=1}^{n} x_{j} \otimes y_{j}$ if and only if the following two conditions hold:

(a) $\sum_{j=1}^{n} u_{j} v_{j}=\left(1-|z|^{2}\right)^{2} \sum_{j=1}^{n} x_{j} \overline{y_{j}}$;

(b) $\sum_{j=1}^{n}\left[\overline{P \overline{u_{j}}}-u_{j}(0)\right]\left[P v_{j}-v_{j}(0)\right]=0$.

\section{Semicommutators of Block \\ Toeplitz Operators}

In this section, we discuss the finite rank semicommutators and commutators of the block Toeplitz operators $T_{F}, T_{G}$ on $L_{a}^{2}\left(\mathbb{D}, \mathbb{C}^{n}\right)$ with $F, G \in h^{\infty} \otimes M_{n \times n}$. In the following part of this paper, $E_{j}$ denote the matrix unit with $(j, j)$ th entry equal to one and all others equal to zero.

Theorem 3. Let $F(z)=F_{+}(z)+F_{-}(z)$, and let $G(z)=G_{+}(z)+$ $G_{-}(z) \in h^{\infty} \otimes M_{n \times n}$ such that $F_{+}(0)=G_{-}(0)=0_{n \times n}$. Then the following statements are equivalent:

(1) $T_{F G}-T_{F} T_{G}$ has finite rank;

(2) $T_{F G}=T_{F} T_{G}$;

(3) for each $1 \leq j \leq n$, there exist a matrix $M_{j} \in M_{n \times n}$ and a permutation matrix $Q_{j}$, such that $M_{j} Q_{j} G_{-} E_{j}=0_{n \times n}$ and $F_{+} Q_{j}\left(I+M_{j}\right)=0_{n \times n}$.

Proof. (1) $\Rightarrow$ (2) Note that $T_{F G}-T_{F} T_{G}=H_{F^{*}}^{*} H_{G}$. If $H_{F^{*}}^{*} H_{G}$ has a finite rank, then $\sum_{p=1}^{n} H_{f_{i p}}^{*} H_{g_{p j}}$ has a finite rank for $1 \leq$ $i, j \leq n$. By Theorem 1 , we have that $\sum_{p=1}^{n} H_{f_{i p}}^{*} H_{g_{p j}}=0$ for $1 \leq i, j \leq n$. Hence, $T_{F G}=T_{F} T_{G}$.

$(2) \Rightarrow$ (3) If $T_{F G}-T_{F} T_{G}=H_{F^{*}}^{*} H_{G}=0$, then $\left[H_{F^{*}}^{*} H_{G}\right]_{i, j}=$ $\sum_{p=1}^{n} H_{f_{i p}}^{*} H_{g_{p j}}=0$, for $1 \leq i, j \leq n$. By Theorem 1 , we get $\left(1-|z|^{2}\right)^{2} \sum_{p=1}^{n}\left[\left(f_{i p}\right)_{+}^{\prime}\left(g_{p j}\right)_{-}^{\prime}\right]=0$ on $\mathbb{D}$. Therefore, $\sum_{p=1}^{n}\left[\left(f_{i p}\right)_{+}^{\prime}\left(g_{p j}\right)_{-}^{\prime}\right]=0$ on $\mathbb{D}$ for $1 \leq i, j \leq n$.

It is known that if $H(z, w)$ is holomorphic in $\mathbb{D} \times \mathbb{D}$ and $H(z, \bar{z})=0$, then $H(z, w) \equiv 0$ for $(z, w) \in \mathbb{D} \times \mathbb{D}$ in several complex variables. So we can "complexificate" the above identity and have $\sum_{p=1}^{n}\left[\left(f_{i p}\right)_{+}^{\prime}(z)\left(g_{p j}\right)_{-}^{\prime}(w)\right]=0$ for $1 \leq i, j \leq n,(z, w) \in \mathbb{D} \times \mathbb{D}$. Therefore,

$$
\begin{aligned}
0 & =\sum_{p=1}^{n}\left[\left(f_{i p}\right)_{+}^{\prime}(u)\left(g_{p j}\right)_{-}^{\prime}(v)\right] \\
& =\int_{0}^{z} \sum_{p=1}^{n}\left[\left(f_{i p}\right)_{+}^{\prime}(u)\left(g_{p j}\right)_{-}^{\prime}(v)\right] d u \\
& =\sum_{p=1}^{n}\left[\left(f_{i p}\right)_{+}(z)\left(g_{p j}\right)_{-}^{\prime}(v)\right] \\
& =\int_{0}^{w} \sum_{p=1}^{n}\left[\left(f_{i p}\right)_{+}(z)\left(g_{p j}\right)_{-}^{\prime}(v)\right] d v \\
& =\sum_{p=1}^{n}\left[\left(f_{i p}\right)_{+}(z)\left(g_{p j}\right)_{-}(w)\right],
\end{aligned}
$$

which means that $F_{+}(z) G_{-}(w)=0_{n \times n}$ on $\mathbb{D} \times \mathbb{D}$.
Note that $\operatorname{det} F_{+}(z)$ is the analytic function of $z$ and $\operatorname{det} G_{-}(w)$ is the coanalytic function of $w$. If $\operatorname{det} F_{+}(z)$ is not identically zero, then $G_{-}=0_{n \times n}$; that is, $G$ is analytic. If $\operatorname{det} G_{-}(w)$ is not identically zero, then $F_{+}=0_{n \times n}$; that is, $F$ is coanalytic.

In the following, we deal with the case that $\operatorname{det} F_{+} \equiv 0$ and det $G_{-} \equiv 0$. Suppose $G_{-} E_{j}=\left(g_{1}^{j}, \ldots, g_{n}^{j}\right)^{T}$ for $1 \leq j \leq n$. Since det $G_{-} \equiv 0, G_{-} E_{j}$ is linearly dependent. Let $Q_{j}$ be a permutation matrix such that

$$
Q_{j} G_{-} E_{j}=\left(g_{\sigma(1)}^{j}, \ldots, g_{\sigma\left(k_{0}\right)}^{j}, \ldots, g_{\sigma(n)}^{j}\right)^{T}
$$

where $\left\{g_{\sigma(1)}^{j}, \ldots, g_{\sigma\left(k_{0}\right)}^{j}\right\}$ is maximally linearly independent of $\left\{g_{\sigma(1)}^{j}, \ldots, g_{\sigma(n)}^{j}\right\}$. And

$$
\begin{aligned}
g_{\sigma\left(k_{0}+1\right)}^{j} & =a_{1}^{1} g_{\sigma(1)}^{j}+\cdots+a_{k_{0}}^{1} g_{\sigma\left(k_{0}\right)}^{j}, \\
& \vdots \\
g_{\sigma(n)}^{j} & =a_{1}^{n-k_{0}} g_{\sigma(1)}^{j}+\cdots+a_{k_{0}}^{n-k_{0}} g_{\sigma\left(k_{0}\right)}^{j},
\end{aligned}
$$

with the matrix representation

$$
\left[\begin{array}{cccccc}
0 & \cdots & 0 & 0 & \cdots & 0 \\
\vdots & & \vdots & \vdots & & \vdots \\
0 & \cdots & 0 & 0 & \cdots & 0 \\
a_{1}^{1} & \cdots & a_{k_{0}}^{1} & -1 & \cdots & 0 \\
\vdots & & \vdots & \vdots & & \vdots \\
a_{1}^{n-k_{0}} & \cdots & a_{k_{0}}^{n-k_{0}} & 0 & \cdots & -1
\end{array}\right]\left[\begin{array}{c}
g_{\sigma(1)}^{j} \\
\vdots \\
g_{\sigma\left(k_{0}\right)}^{j} \\
\vdots \\
g_{\sigma(n-1)}^{j} \\
g_{\sigma(n)}^{j}
\end{array}\right]=0_{n \times n},
$$

that is, $M_{j} Q_{j} G_{-} E_{j}=0_{n \times n}$.

Since $F_{+}(z)\left[G_{-}(w) E_{j}\right]=0$, we have $\left[F_{+}(z) Q_{j}\right]\left[Q_{j} G_{-}\right.$ $\left.\times(w) E_{j}\right]=0_{n \times n}$. Denote $F_{+}=\left[f_{i j}\right]_{n \times n}$. It follows that

$$
f_{k \sigma(1)} g_{\sigma(1)}^{j}+\cdots+f_{k \sigma\left(k_{0}\right)} g_{\sigma\left(k_{0}\right)}^{j}+\cdots+f_{k \sigma(n)} g_{\sigma(n)}^{j}=0
$$

for $1 \leq k \leq n$. Note that $g_{\sigma\left(k_{0}+l\right)}^{j}=a_{1}^{l} g_{\sigma(1)}^{j}+\cdots+a_{k_{0}}^{l} g_{\sigma\left(k_{0}\right)}^{j}$, $1 \leq l \leq n-k_{0}$. Therefore

$$
\begin{aligned}
f_{k \sigma(1)} & g_{\sigma(1)}^{j}+\cdots+f_{k \sigma\left(k_{0}\right)} g_{\sigma\left(k_{0}\right)}^{j} \\
& +\sum_{l=1}^{n-k_{0}}\left(a_{1}^{l} g_{\sigma(1)}^{j}+\cdots+a_{k_{0}}^{l} g_{\sigma\left(k_{0}\right)}^{j}\right) f_{k \sigma\left(k_{0}+l\right)}=0,
\end{aligned}
$$


and hence, $\left(f_{k \sigma(1)}+\sum_{l=1}^{n-k_{0}} a_{1}^{l} f_{k \sigma\left(k_{0}+l\right)}\right) g_{\sigma(1)}^{j}+\left(f_{k \sigma(2)}+\sum_{l=1}^{n-k_{0}} a_{2}^{l}\right.$ $\left.\times f_{k \sigma\left(k_{0}+l\right)}\right) g_{\sigma(2)}^{j}+\cdots+\left(f_{k \sigma\left(k_{0}\right)}+\sum_{l=1}^{n-k_{0}} a_{k_{0}}^{l} f_{k \sigma\left(k_{0}+l\right)}\right) g_{\sigma\left(k_{0}\right)}^{j}=0$. Since $\left\{g_{\sigma(1)}^{j}, \ldots, g_{\sigma\left(k_{0}\right)}^{j}\right\}$ is linearly independent, we have

$$
\begin{gathered}
f_{k \sigma(1)}+\sum_{l=1}^{n-k_{0}} a_{1}^{l} f_{k \sigma\left(k_{0}+l\right)}=0, \\
\vdots \\
f_{k \sigma\left(k_{0}\right)}+\sum_{l=1}^{n-k_{0}} a_{k_{0}}^{l} f_{k \sigma\left(k_{0}+l\right)}=0,
\end{gathered}
$$

with the matrix representation

$$
\begin{gathered}
{\left[\begin{array}{ccccc}
f_{1 \sigma(1)} & \cdots & f_{1 \sigma\left(k_{0}\right)} & \cdots & f_{1 \sigma(n)} \\
f_{2 \sigma(1)} & \cdots & f_{2 \sigma\left(k_{0}\right)} & \cdots & f_{2 \sigma(n)} \\
\vdots & & \vdots & & \vdots \\
\vdots & & \vdots & & \vdots \\
f_{n-1 \sigma(1)} & \cdots & f_{n-1 \sigma\left(k_{0}\right)} & \cdots & f_{n-1 \sigma(n)} \\
f_{n \sigma(1)} & \cdots & f_{n \sigma\left(k_{0}\right)} & \cdots & f_{n \sigma(n)}
\end{array}\right]} \\
\quad\left[\begin{array}{cccccc}
1 & \cdots & 0 & 0 & \cdots & 0 \\
\vdots & & \vdots & \vdots & & \vdots \\
0 & \cdots & 1 & 0 & \cdots & 0 \\
a_{1}^{1} & \cdots & a_{k_{0}}^{1} & 0 & \cdots & 0 \\
\vdots & & \vdots & \vdots & & \vdots \\
a_{1}^{n-k_{0}} & \cdots & a_{k_{0}}^{n-k_{0}} & 0 & \cdots & 0
\end{array}\right]=0_{n \times n},
\end{gathered}
$$

that is $F_{+} Q_{j}\left(I+M_{j}\right)=0_{n \times n}$.

In the following, we prove (3) $\Rightarrow(1)$.

Note that

$$
\begin{aligned}
T_{F} T_{G}-T_{F G}= & T_{\left(F_{+}+F_{-}\right)} T_{\left(G_{+}+G_{-}\right)}-T_{\left(F_{+}+F_{-}\right)\left(G_{+}+G_{-}\right)} \\
= & T_{\left(F_{+} G_{+}+F_{-} G_{-}+F_{-} G_{+}\right)}+T_{F_{+} G_{-}} \\
& -T_{\left(F_{+} G_{+}+F_{-} G_{-}+F_{-} G_{+}\right)}-T_{F_{+} G_{-}} \\
= & T_{F_{+}} T_{G_{-}}-T_{F_{+} G_{-}} .
\end{aligned}
$$

By the hypothesis, we have

$$
\begin{aligned}
T_{F_{+}} T_{G_{-} E_{j}}= & T_{F_{+} Q_{j}\left(I+M_{j}-M_{j}\right) Q_{j}} T_{G_{-} E_{j}} \\
= & T_{F_{+} Q_{j}\left(I+M_{j}\right) Q_{j}} T_{G_{-} E_{j}}-T_{F_{+} Q_{j} M_{j} Q_{j}} T_{G_{-} E_{j}} \\
= & T_{F_{+} Q_{j}\left(I+M_{j}\right) Q_{j}} T_{G_{-} E_{j}}-T_{F_{+} Q_{j}} T_{M_{j} Q_{j} G_{-} E_{j}}=0_{n \times n}, \\
F_{+} G_{-} E_{j}= & F_{+} Q_{j}\left[I+M_{j}-M_{j}\right] Q_{j} G_{-} E_{j} \\
= & {\left[F_{+} Q_{j}\left(I+M_{j}\right)\right] Q_{j} G_{-} E_{j} } \\
& -F_{+} Q_{j}\left[M_{j} Q_{j} G_{-} E_{j}\right]=0_{n \times n} .
\end{aligned}
$$

Hence, we obtain the desired result.
Remark 4. If the assumption $F_{+}(0)=G_{-}(0)=0_{n \times n}$ is removed and $F_{+}, G_{-}$are replaced by $F_{+}^{\prime}, G_{-}^{\prime}$ in statement (3), respectively, then the above theorem still holds.

Corollary 5. Let $F(z)=F_{+}(z)+F_{-}(z)$, and let $G(z)=$ $G_{+}(z)+G_{-}(z) \in h^{\infty} \otimes M_{n \times n}$ such that $F_{+}(0)=G_{-}(0)=0_{n \times n}$. Suppose that $\left|\operatorname{det} F_{+}\right|+\left|\operatorname{det} G_{-}\right|$is not identically zero. Then the following are equivalent:

(1) $T_{F G}-T_{F} T_{G}$ has finite rank;

(2) $T_{F G}=T_{F} T_{G}$;

(3) if $\operatorname{det} F_{+}$is not identically zero, then $G$ is analytic; if $\operatorname{det} G_{-}$is not identically zero, then $F$ is coanalytic.

Proof. The proof is obvious.

Let

$$
\Phi=\left[\begin{array}{cc}
F & -G \\
0 & 0
\end{array}\right], \quad \Psi=\left[\begin{array}{cc}
G & 0 \\
F & 0
\end{array}\right] .
$$

As in [6], the commutator $T_{F} T_{G}-T_{G} T_{F}$ can be reduced to the semicommutator $T_{\Phi \Psi}-T_{\Phi} T_{\Psi}$.

It is easy to check that

$$
\begin{aligned}
T_{\Phi \Psi} & -T_{\Phi} T_{\Psi} \\
& =\left[\begin{array}{cc}
T_{F G-G F} & 0 \\
0 & 0
\end{array}\right]-\left[\begin{array}{cc}
T_{F} & -T_{G} \\
0 & 0
\end{array}\right]\left[\begin{array}{ll}
T_{G} & 0 \\
T_{F} & 0
\end{array}\right] \\
& =\left[\begin{array}{cc}
T_{F G-G F} & 0 \\
0 & 0
\end{array}\right]-\left[\begin{array}{cc}
T_{F} T_{G}-T_{G} T_{F} & 0 \\
0 & 0
\end{array}\right] .
\end{aligned}
$$

If $T_{F} T_{G}=T_{G} T_{F}$, Theorem 2 implies that $F G=G F$. Therefore, $T_{\Phi} T_{\Psi}=T_{\Psi \Phi}$. Combining Theorem 3, we get the following explicit theorem.

Theorem 6. Suppose that $F=F_{+}+F_{-} \in h^{\infty} \otimes M_{n \times n}$ and $G=G_{+}+G_{-} \in h^{\infty} \otimes M_{n \times n}$. Then $T_{F} T_{G}=T_{G} T_{F}$ if and only if $F$ and $G$ satisfy the following two conditions:

(1) $F G=G F$

(2) for each $1 \leq j \leq n$, there exist permutation matrixes $\widetilde{Q_{j}}$ and $\mathbb{M}_{j} \in M_{2 n \times 2 n}$ such that $\mathbb{M}_{j} \widetilde{Q_{j}}\left[\begin{array}{c}G_{-}^{\prime} \\ F_{-}^{\prime}\end{array}\right] E_{j}=0_{n \times n}$ and $\left[F_{+}^{\prime}-G_{+}^{\prime}\right] \widetilde{Q_{j}}\left(I+\mathbb{M}_{j}\right)=0_{n \times n}$.

\section{Zero Product of Two Block Toeplitz Operators}

In this section, we discuss the zero product of two block Toeplitz operators $T_{F}$ and $T_{G}$ with $F, G \in h^{\infty} \otimes M_{n \times n}$ on $L_{a}^{2}\left(\mathbb{D}, \mathbb{C}^{n}\right)$. 
Theorem 7. Let $F(z)=F_{+}(z)+F_{-}(z)$ and $G(z)=G_{+}(z)+$ $G_{-}(z) \in h^{\infty} \otimes M_{n \times n}$ such that $F_{+}(0)=G_{-}(0)=F_{-}(0)=$ $G_{+}(0)=0_{n \times n}$. Then $T_{F} T_{G}=0_{n \times n}$ if and only if

(1) $F G=0_{n \times n}$;

(2) for each $1 \leq j \leq n$, there exist a matrix $N_{j} \in M_{n \times n}$ and a permutation matrix $Q_{j}^{1}$, such that $N_{j} Q_{j}^{1} G_{-} E_{j}=0_{n \times n}$ and $F_{+} Q_{j}^{1}\left(I+N_{j}\right)=0_{n \times n}$.

Proof. By Theorem 2, it is easy to know that $\sum_{p=1}^{n} f_{i p}(z) g_{p j}(z)=0$ for $1 \leq i, j \leq n$; that is, $F G=0_{n \times n}$. Since $T_{F} T_{G}=T_{F} T_{G}-T_{F G}=0_{n \times n}$, we obtain the desired result by Theorem 3 .

Corollary 8. Let $F(z)=F_{+}(z)+F_{-}(z)$ and let $G(z)=G_{+}(z)+$ $G_{-}(z) \in h^{\infty} \otimes M_{n \times n}$ such that $F_{+}(0)=G_{-}(0)=F_{-}(0)=$ $G_{+}(0)=0_{n \times n}$.

(1) If neither $\operatorname{det} G_{+}$nor $\operatorname{det} G_{-}$is identically zero, then $T_{F} T_{G}=0$ if and only if $F=0_{n \times n}$.

(2) If neither det $F_{+}$nor det $F_{-}$is identically zero, then $T_{F} T_{G}=0$ if and only if $G=0_{n \times n}$.

Proof. (1) We only need to prove the necessity. By the proof of Theorem 3, we have $F_{+}(z) G_{-}(w)=0_{n \times n}$ on $\mathbb{D} \times \mathbb{D}$. By the theorem given above, we know that $F G=\left[F_{+}+F_{-}\right]\left[G_{+}+G_{-}\right]=$ $0_{n \times n}$. Taking Laplace transform, it follows that $F_{-}^{\prime} G_{+}^{\prime}=0_{n \times n}$. As in Theorem 3, we can prove that $F_{-}(w) G_{+}(z)=0_{n \times n}$ on $\mathbb{D} \times \mathbb{D}$. "Complexificate" the identity $F G=0_{n \times n}$, and then $\left[F_{+}(z)+F_{-}(w)\right]\left[G_{+}(z)+G_{-}(w)\right]=0_{n \times n}$. Hence, $F_{+}(z) G_{+}(z)=$ $-F_{-}(w) G_{-}(w)$ on $\mathbb{D} \times \mathbb{D}$. Since $F_{+}(0)=G_{-}(0)=0_{n \times n}$, we have $F_{+} G_{+}=F_{-} G_{-}=0_{n \times n}$.

It is clear that det $G_{+}(z)$ is the analytic function of $z$ and $\operatorname{det} G_{-}(w)$ is the coanalytic function of $w$. If $\operatorname{det} G_{+}(z)$ is not identically zero, then $F_{-} \equiv 0_{n \times n}$ and $F_{+} \equiv 0_{n \times n}$. Therefore, $F=0_{n \times n}$. If det $G_{-}(w)$ is not identically zero, then $F_{-} \equiv 0_{n \times n}$ and $F_{+} \equiv 0_{n \times n}$. Therefore, $F=0_{n \times n}$.

(2) The proof is similar to the proof of (1).

\section{Acknowledgments}

This research is supported by NSFC (11271059, 11201438), Research Fund for the Doctoral Program of Higher Education of China, Shandong Province Young Scientist Research Award Fund (BS2012SF031), and the Fundamental Research Funds for the Central Universities (201213011).

\section{References}

[1] S. Axler, S. Y. A. Chang, and D. Sarason, "Products of Toeplitz operators," Integral Equations and Operator Theory, vol. 1, no. 3, pp. 285-309, 1978.

[2] X. Ding and D. Zheng, "Finite rank commutator of Toeplitz operators or Hankel operators," Houston Journal of Mathematics, vol. 34, no. 4, pp. 1099-1119, 2008.

[3] P. Ahern and Ž. Čučković, "A theorem of Brown-Halmos type for Bergman space Toeplitz operators," Journal of Functional Analysis, vol. 187, no. 1, pp. 200-210, 2001.
[4] S. Axler and Ž. Čučković, "Commuting Toeplitz operators with harmonic symbols," Integral Equations and Operator Theory, vol. 14, no. 1, pp. 1-12, 1991.

[5] D. C. Zheng, "Hankel operators and Toeplitz operators on the Bergman space," Journal of Functional Analysis, vol. 83, no. 1, pp. 98-120, 1989.

[6] C. Gu and D. Zheng, "Products of block Toeplitz operators," Pacific Journal of Mathematics, vol. 185, no. 1, pp. 115-148, 1998.

[7] K. Guo, S. Sun, and D. Zheng, "Finite rank commutators and semicommutators of Toeplitz operators with harmonic symbols," Illinois Journal of Mathematics, vol. 51, no. 2, pp. 583596, 2007.

[8] B. R. Choe, H. Koo, and Y. J. Lee, "Sums of Toeplitz products with harmonic symbols," Revista Mathemática Iberoamericana, vol. 24, no. 1, pp. 43-70, 2008. 


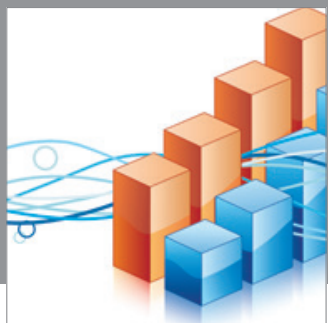

Advances in

Operations Research

mansans

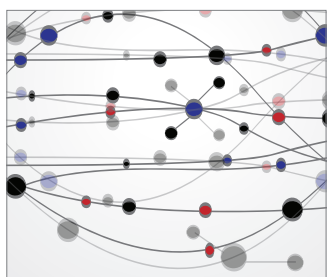

The Scientific World Journal
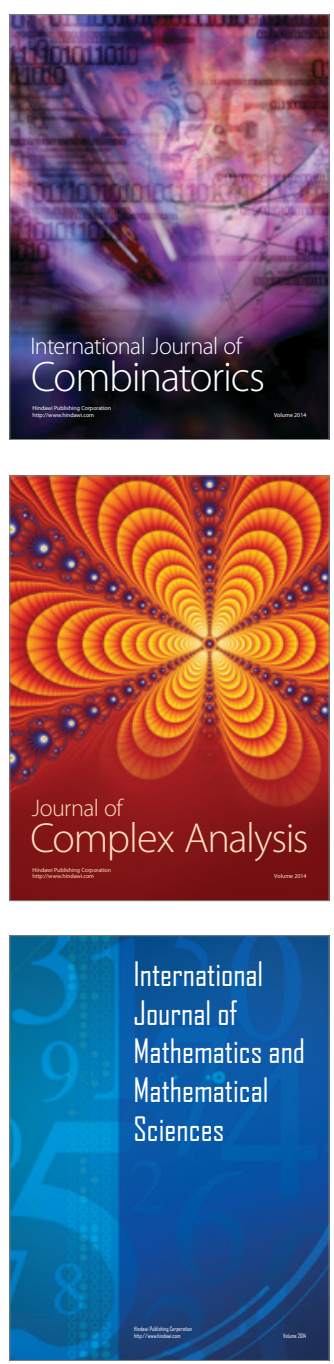
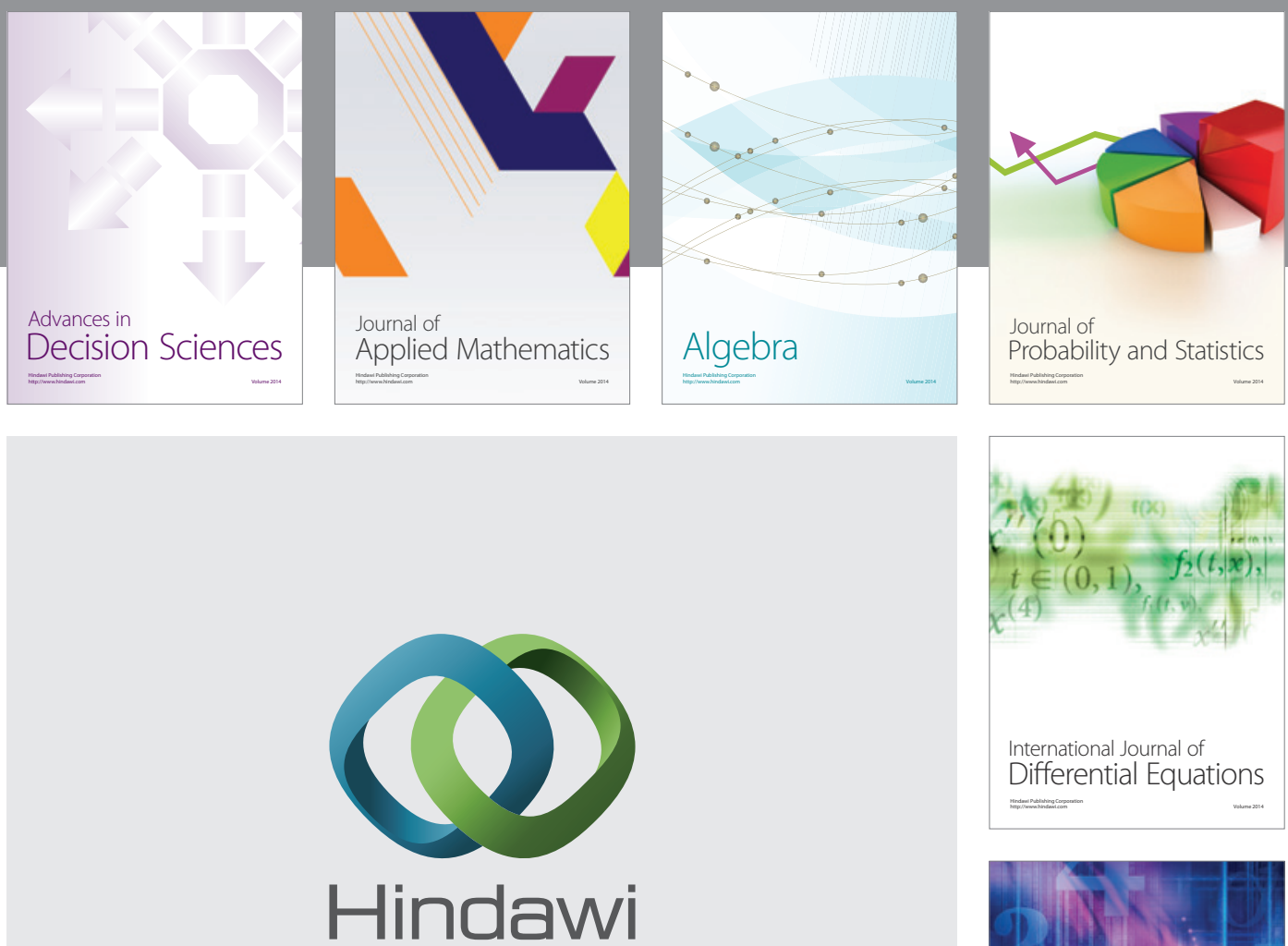

Submit your manuscripts at http://www.hindawi.com
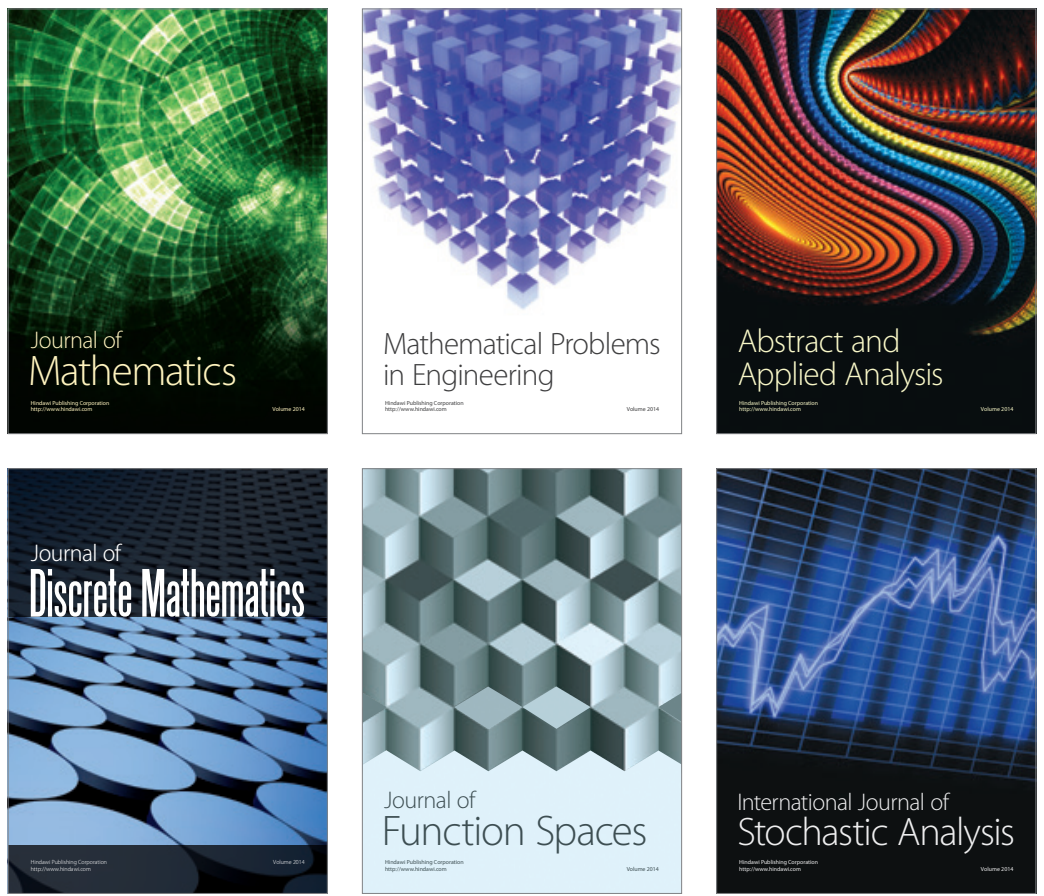

Journal of

Function Spaces

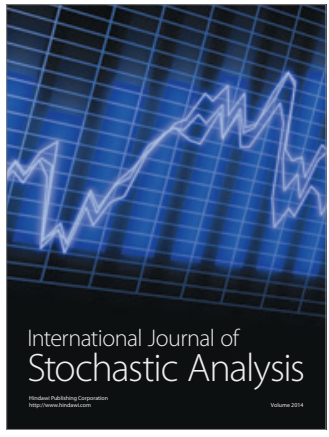

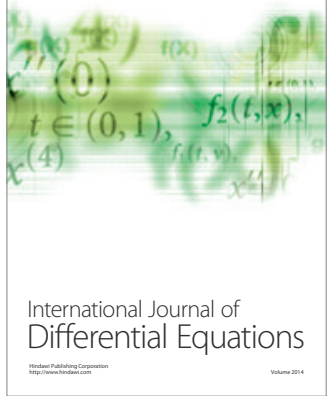
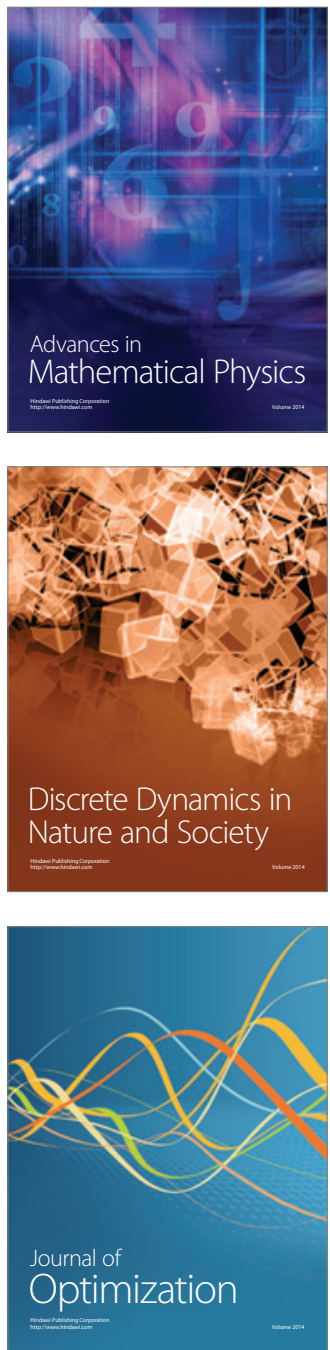